FORMATION Formation emploi

Revue française de sciences sociales

107 | juillet-septembre 2009

La formation professionnelle en Amérique latine

\title{
Associations d'aide à domicile : pluralité des héritages, pluralité des professionnalités
}

Asociaciones de ayuda domiciliaria: pluralidad de herencias, pluralidad de profesionalidades

Vereine zur Haushaltshilfe: Viele Hinterlassenschaften, viele Professionalitäten Home-care associations: diverse backgrounds; diverse professional skills

Florence Jany-Catrice, Emmanuelle Puissant et Thierry Ribault

\section{OpenEdition}

Journals

Édition électronique

URL : http://journals.openedition.org/formationemploi/2026

DOI : 10.4000/formationemploi.2026

ISSN : 2107-0946

Éditeur

La Documentation française

Édition imprimée

Date de publication : 1 septembre 2009

Pagination : $77-91$

ISSN : 0759-6340

Référence électronique

Florence Jany-Catrice, Emmanuelle Puissant et Thierry Ribault, « Associations d'aide à domicile : pluralité des héritages, pluralité des professionnalités », Formation emploi [En ligne], 107 | juilletseptembre 2009, mis en ligne le 01 septembre 2011, consulté le 30 octobre 2020. URL : http:// journals.openedition.org/formationemploi/2026 ; DOI : https://doi.org/10.4000/formationemploi.2026

(c) Tous droits réservés 


\title{
Profession
}

\section{Associations d'aide à domicile : pluralité des héritages, pluralité des professionnalités 1}

Florence Jany-Catrice, Emmanuelle Puissant, Thierry Ribault ${ }^{\star}$

\author{
Réduire la professionnalisation à un processus d'individualisation \\ de la qualification des salariés, ou à une démarche de rationalisation \\ des prestations, brouille la représentation des tâches. La pluralité des formes \\ $d^{\prime}$ engagement des intervenantes à domicile constitue précisément \\ une caractéristique majeure du secteur.
}

La professionnalisation est, de façon assez consensuelle, présentée comme une solution miraculeuse à une partie des maux relatifs aux emplois des aides à domicile aux personnes fragiles (aide aux personnes âgées, personnes handicapées et enfants), traditionnellement portés par le monde associatif. Elle mènerait à de meilleures conditions d'emploi et de travail, à une reconnaissance économique et sociale et à une meilleure qualité de service.

Pourquoi dans ce cas tarder à professionnaliser ? Peuton réduire la professionnalisation dans ces services à une vision industrialiste, marchande et individualisée de la professionnalité ? Dans quelles mesures les

\footnotetext{
${ }^{1}$ Le présent article est issu d'une contribution pour le colloque «Économie sociale et solidaire : nouvelles pratiques et dynamiques territoriales », qui s'est déroulé à Nantes les 29 et 30 septembre 2008. Il propose une mise en dialogue des résultats des travaux de terrain mentionnés et se situe, d'un point de vue disciplinaire, au carrefour de deux types d'approches : la socio-économie des services et l'économie sociale et solidaire. Cet article est le fruit de la rencontre de deux travaux : (voir encadré méthodologique).
}

services rendus renvoient-ils à une qualification collective et construite ? Quelles sont les conditions d'élaboration de cette construction collective ?

* Florence Jany-Catrice est économiste, CLERSÉ(Centre Lillois d'études et de recherches sociologiques et économiques) UMR 8019, université Lille 1.

Florence.jany-catrice@univ-lillel.fr

Axes de recherches: socio-économie du travail et de l'emploi dans les services; recomposition des emplois non qualifiés; évaluation des performances et de la richesse dans des économies de la "qualité ".

Elle a publié récemment : 2009, avec Devetter F.-X. et Ribault T., Les services à la personne, La Découverte, coll. "Repères », 128 p. 2007, avec Gadrey J., (réed.), Les nouveaux indicateurs de richesse, La Découverte, coll. "Repères », $128 \mathrm{p}$. 
Emmanuelle Puissant est doctorante en sciences économiques, Laboratoire CREPPEM /Centre de recherche économique sur les politiques publiques dans une économie de marché), ESEAC léquipe de socio-économie associative et coopérative)-IEP (Institut d'études politiques), université Grenoble 2.

emmanuelle.puissant@wanadoo.fr

Axes de recherche : économie sociale et solidaire, socioéconomie du travail, socio-économie des services.

Publications récentes : 2008, avec Artis A., Demoustier D., Hofmann B., Économie sociale et solidaire et régulations territoriales, Rapport pour la Délégation Interministérielle à I'Innovation, l'Expérimentation sociale et à l'Économie sociale. 2007 " Associations d'aide à domicile : des tensions à l'œeuvre dans la relation de travail », Contribution au colloque annuel de l'Association d'économie sociale, Paris. economix.u-paris 10.fr/pdf/colloques/2007 AES/puissant.pdf

Thierry Ribault est économiste, chercheur au CNRS, Laboratoire CLERSÉ, UMR 8019 Villeneuve d'Ascq

thierry.ribault@ifresi.univ-lille].fr

Principaux axes de recherches : I'emploi et le travail dans les services. Comparaisons France-Japon.

Il a publié récemment : 2009, avec Devetter F.-X. et JanyCatrice F., Les services à la personne, La Découverte, collection "Repères», Paris. 2008. "Aide à domicile: de l'idéologie de la professionnalisation à la pluralité des professionnalités », Revue Française de Socio-Économie $n^{\circ} 2$.

Nous proposons, à partir de travaux empiriques, d'analyser ces nouveaux enjeux, en raisonnant en trois temps. Dans un premier temps, nous revenons sur la notion de professionnalisation, dont l'usage dans les discours et les pratiques s'accroît tout en s'individualisant à mesure que la tertiarisation des économies progresse. Dans un second temps, nous montrons en quoi l'héritage de l'économie sociale rend complexe et tendu le rapport à la professionnalisation des aides à domicile, telle qu'elle s'opère dans ce climat d'individualisation. Enfin, nous identifions une pluralité de conventions de professionnalité, souvent imbriquées, concrètement à l'œuvre dans ce champ, et dont la reconnaissance peut représenter une opportunité que les acteurs de l'économie sociale et solidaire (ESS) sont le mieux à même de saisir.

\section{QUALIFIER OU PROFESSIONNALISER ?}

Revenir sur la notion de professionnalisation nécessite d'abord une mise en perspective analytique visà-vis de la notion de qualification. Dans un second temps, nous en proposerons une critique à l'aune des pratiques observées dans l'aide à domicile.

\section{Deux notions en divorce}

\section{Non qualification et professionnalisation}

Dans le registre statistique, un consensus classe les aides à domicile parmi les employées «non qualifiées » (Gadrey et alii, 2004), soit parce que leur salaire est faible au regard des pratiques salariales intersectorielles (Burnod, Chenu, 2001), soit que la part des diplômés est réduite (Chardon, 2002), soit encore que les conventions collectives identifient peu d'autonomie, de responsabilité et d'initiatives dans l'exercice du travail (Bisault et alii, 1994).

Les représentations des exigences attendues - dont nos travaux de terrain rendent compte (voir encadré 1) - insistent pourtant sur la nécessité d'une professionnalisation. Le vieillissement de la population exigerait de manière croissante des compétences de professionnels accompagnant les personnes âgées, « n’importe qui » n’étant pas en mesure de s'occuper des personnes en situation de fragilité. La professionnalisation du champ de l'aide à domicile conditionnerait la qualité du service rendu.

Il y a donc une tension entre des catégories «non qualifiées » d'un côté et une "exigence de professionnalisation " de l'autre, la première renvoyant plutôt à des conventions d'ordre statistique, la seconde relevant plutôt de la convention des représentations - ordinaires ou spécifiques - du métier $^{2}$ de l'aide à domicile.

La définition de la qualification des employés est donc en jeu (Méda, Vennat, 2004). Dans les métiers à

\footnotetext{
2 On considérera ici que la « profession » est le résultat du processus de reconnaissance du «métier » entendu comme la maîtrise d'un ensemble d'aptitudes qui permet de répondre à un besoin donné.
} 


\section{Encadré 1 \\ Méthodologie}

L'analyse repose sur l'exploitation et l'usage de plusieurs types de matériaux. II s'agit (1) des statistiques du champ des services à la personne à partir des données administratives Ircem (Caisse de retraite des emplois du particulier employeur) et Dares (Direction de l'animation de la recherche, des études et des statistiques) (pour les organismes agréés des services à la personne) ; (2) des textes officiels régulant l'activité et le secteur d'activité laccords de branche, loi du 2 janvier 2002, informations de propagande de l'agence nationale des services à la personne) ; (3) des principales conventions collectives.

Ces données qualitatives et quantitatives ont été complétées de deux manières. D'une part, par des monographies d'associations d'aide à domicile dans le département de l'Isère, sur la base d'une immersion de plusieurs mois dans une association mandataire et prestataire, et de 60 entretiens semi-directifs et ouverts menés en 2006 (Puissant, 2008). D'autre part, dans le cadre d'une recherche réalisée pour la Diieses, (Délégation interministérielle à l'innovation, à l'expérimentation sociale et à l'économie sociale) 85 entretiens semi-directifs, menés sur les départements de Paris, la Seine-Saint-Denis, le Nord, le Pas-de-Calais et la Seine Maritime en 2007, ont été exploités (Devetter, Fraisse, Gardin, Gounouf, Jany-Catrice, Ribault, 2008).

Les monographies et entretiens ont été réalisés auprès d'une pluralité d'acteurs : responsables associatifs (bénévoles et salariés) et privés lucratifs, représentants d'enseignes, des salariés (aides à domicile et encadrants intermédiaires), acteurs de la régulation (Conseils Généraux, DRTEFP, DDTEFP, ANSP Direction régionale du Travail de l'Emploi et de la Formation professionnelle; Direction départementale du Travail, de l'Emploi et de la Formation professionnelle; Agence nationale des services à la personne), représentants prud'homaux employeurs et salariés, usagers. Les thèmes portaient en majorité sur la qualité de l'emploi et la qualité du service, les dispositifs de production de signaux de qualité, et sur les dispositifs de professionnalisation. Des entretiens semi-directifs ont été menés, et ont varié d'une heure à deux heures selon les protagonistes interviewés.

dominante féminine du tertiaire, les critères « traditionnels » - diplôme, salaire, autonomie, responsabilité - sont insuffisants pour opérer une distinction entre «qualifié » et «non-qualifié ». Certes, l'étude des contenus de travail enrichit l'analyse des compétences, et met en évidence des qualités mobilisées par les employés et exigées lors des recrutements. Mais ces qualités sont souvent peu reconnues socialement, notamment parce qu'elles sont considérées comme banales, substituables, ou encore abondamment disponibles (Gadrey et alii, op. cit.).

Or la qualification pas plus que la non-qualification ne résultent d'un équilibre sur un marché où s'échangeraient des caractéristiques clairement identifiées et donc « objectivées » ex-ante. Elle est un véritable construit social où interviennent des rapports de force, des rapports de genre notamment. Cela ne signifie pas que les caractéristiques mobilisées ne sont jamais objectivables, mais que l'objectivation des caractéristiques de qualité s'inscrit précisément dans un processus de négociation autour d'activités aux résultats incertains.

\section{Des débats théoriques aux lourdes implications pratiques}

S’ils traitent le plus souvent de l'existence et de la reconnaissance de la qualification et des compétences, les débats théoriques évoquent rarement le cas des salariés dont la qualification et les compétences sont déniées, en particulier dans les services auprès des personnes fragiles. Les débats sur ce thème se sont traditionnellement organisés autour de deux tendances distinctes. P. Naville (1956) sur le temps de 


\section{Encadré 2}

\section{Les protagonistes de l'aide à domicile}

Depuis l'accord de branche du 29 mars 2002 sur les catégories et les rémunérations, les salariés de l'aide à domicile relèvent de différentes catégories selon le niveau de qualifications. Le titre générique pour les qualifier est celui "d'aide à domicile » qui distingue, conventionnellement, 3 catégories : A, B, C. La catégorie A représente les "agents à domicile», dont l'embauche ne nécessite aucune qualification ni formation. La catégorie B regroupe les «employés à domicile ». Soit les employées à domicile détiennent une qualification (BEP, titre professionnel " assistant de vie » de l'AFPA - Association pour la formation professionnelle des adultes), soit elles sont en cours de validation des acquis de l'expérience pour obtenir le DEAVS (diplôme d'État d'auxiliaire de vie sociale). La catégorie C regroupe les salariées titulaires du DEAVS, qui ont le titre d'« auxiliaire de vie sociale». Enfin, l'aide à domicile aux familles compte une dernière catégorie, la catégorie $B$, qui comprend les techniciens d'intervention sociale et familiale, titulaires du diplôme d'État du même nom.

Les organisations des services d'aide à domicile proviennent historiquement, et encore largement, du secteur associatif. Les associations fonctionnent principalement en mode prestataire /l'association emploie les aides à domicile), mais développent souvent en parallèle un service mandataire (les aides à domicile sont employées par les usagers, mais l'association intervient comme une aide dans la réalisation des tâches administratives). Avec une place quantitative beaucoup plus modeste, les centres communaux d'action sociale sont aussi des acteurs historiques du champ. Si les entreprises privées à but lucratif émergent dans ce secteur, elles constituent, pour l'instant, une part infime dans les activités agréées pour la prise en charge des personnes âgées et dépendantes.

Mais parallèlement à ces organisations structurées et parfois agréées, la plupart de l'offre de services relève d'un régime de gré à gré, dans le cadre duquel l'employeur est aussi l'usager du service. Dans l'ensemble des services à la personne, l'emploi direct représente $85 \%$ des heures. Selon une étude de la Drees (Direction de la recherche des études, de l'évaluation et des statistiques) (Weber, 2006), dans l'aide à domicile financée par l'APA, lallocation personnalisée d'autonomie mise en place en 2002, attribuée par les Conseils généraux, et variant en fonction du niveau de dépendance de la personne âgée) l'emploi direct et le mandataire représentaient, en 2003, 38 \% des prestations financées.

Le secteur a connu une longue série de régulations qui sont venues tout à la fois structurer l'offre de services, subventionner la demande, et inciter à la création d'emplois. Sur le pan de l'action sociale, ce sont les Cram (Caisses régionales d'assurance maladie) pour les personnes les plus légèrement dépendantes, et les Conseils généraux pour les personnes âgées plus dépendantes, qui sont les acteurs majeurs du champ. Le suivi de la politique d'emploi est plutôt assuré par l'ANSP (Agence nationale des services à la personne). Dans un souci de "fluidification" du marché de l'aide à domicile, la loi de développement de la cohésion sociale du 2005, a instauré les enseignes nationales. Ces enseignes regroupent différents acteurs et financeurs du champ (historiques comme les associations, les mutuelles) ou nouvellement entrés sur ce champ (comme des grands groupes commerciaux et financiers), et ont pour rôle l'appariement entre offre et demande de services.

formation nécessaire pour acquérir les connaissances indispensables à l'exercice du métier, et G. Friedmann et P. Naville (1962) appréhendent la qualification à partir du poste de travail. «Dès l'origine donc, le double aspect de la qualification - qualification individuelle ou qualification du poste de travail - est bien analysé, mais l'incertitude qu'il crée dans la recherche de critères pertinents pour définir la 
qualification n'a jamais pu être réduite " (Oiry, d'Iribarne, 2001). Les études sur la construction des grilles de classification ont remis en cause la pertinence d'une approche substantialiste de la qualification et l'ont définie comme un enjeu social, selon une approche plus constructiviste (Gadrey et alii, op. cit).

Ces deux courants ont cohabité dans les recherches sur la qualification, même si l'approche constructiviste a occupé une place prépondérante dans les années 70. L’apparition de la notion de compétence, son corollaire la professionnalisation, et leur large diffusion dans les pratiques de gestion des entreprises, ont toutefois relancé les débats. À la différence de la qualification, la professionnalisation par identification des compétences mobilisées est plutôt liée à un résultat (Reynaud, 2001). La professionnalisation ne s'évalue et ne se valide que dans et par l'entreprise. Il ne s'agit plus de construire, par la négociation et le compromis social, une hiérarchie des qualifications et des salaires, mais bien de mesurer objectivement la compétence, ce qui redonne force aux conceptions substantialistes, et rend quasi caduques à la fois des espaces de négociations, mais aussi l'exigence de règles, normes et conventions construites pour un collectif. Le succès de la notion de « validation des acquis de l'expérience » s'inscrit en partie dans ce revirement ${ }^{3}$. Les exigences de professionnalisation peuvent s'interpréter comme un aveu d'échec des processus de qualification, une manière de contourner les exigences de réconciliation des trois pôles de la qualification : qualifications individuelle, du poste du travail, et salariale (Rose, 2004). Mais la professionnalisation est une notion qui n'est pas elle-même dénuée d'ambiguïtés. Elle peut renvoyer à l'idée de professionnalisation comme communauté semi-fermée. M. Lallement (2007) identifie les critères, consensuels chez les

\footnotetext{
${ }^{3}$ Historiquement, les acteurs de l'ESS ont impulsé des dynamiques de qualifications collectives, assorties de l'obtention de droits du travail et sociaux collectifs (Artis et alii, 2008). Depuis les années 90, les choix publics ont toutefois contraint les organisations d'ESS à limiter de telles pratiques. Dans l'aide à domicile, notamment depuis 2002 avec l'instauration du droit individuel de la Validation des acquis de l'expérience (VAE), les organisations d'ESS ont tendance à privilégier une logique de qualification individuelle des personnes, sur la base de reconnaissance des compétences acquises au cours de leur expérience professionnelle, au détriment d'une logique de qualifications collectives des emplois, sur la base d'apprentissages de savoirs nouveaux.
}

sociologues fonctionnalistes, selon lesquels les professions ont avantage à fonctionner comme une communauté, à ne pas ouvrir grandes les portes qui mènent à elles et à maintenir élevée la qualité des services rendus ${ }^{4}$.

L'acception qui tend à s'imposer dans le métier de l'aide à domicile est donc celle d'un processus d'individualisation des qualifications et d'identification des compétences. La professionnalisation de l'aide à domicile se voit ainsi attribuer le rôle d'une nouvelle idéologie plutôt que celui d'un «espace professionnel négocié» où le bien-être de la personne bénéficiaire du service et les conditions de travail et d'emploi de celle qui le dispense seraient conçus conjointement et de manière dynamique (Jany-Catrice, Ribault, 2007 ; Ribault 2007). Le caractère intime de la relation de service vient légitimer un attachement des compétences à la personne du travailleur, voire à ses qualités personnelles.

Nous prenons le parti d'une approche (macro-)interactionniste selon laquelle la question est moins de savoir si telle occupation est ou non une profession, mais plutôt de s'interroger sur les conditions qui permettent à une activité - ici l'aide à domicile - de s'imposer comme profession (Vasselin, 2002). Produit de déterminants et d'interactions multiples, les professions se structurent et évoluent sous la pression de sous-groupes en opposition, et de tendances parfois contradictoires.

Dans l'aide à domicile, comme pour d'autres catégories de salariés classés comme «non qualifiés », s'observent des distorsions de plus en plus fortes entre qualifications individuelle, de l'emploi, et salariale. Mais peut-on réduire la professionnalisation dans les services d'aide à domicile à une vision industrialiste, marchande et individualisée de la professionnalité ? Dans quelles mesures les services rendus, encore souvent considérés comme une externalisation de tâches domestiques, peuvent-ils ou doivent-ils renvoyer à une qualification collective et construite? 
Reposer les enjeux théoriques

à la lumière de l'observation des pratiques

Parler d' «industrialisation » pour qualifier les évolutions en cours dans les activités de service n'est pas nouveau (Ughetto, 2002). Toutefois, ce processus concerne plutôt des activités de services qui ne se réalisent pas en continu en présence des usagers. L'industrialisation émergente dans l'aide à domicile, vecteur puissant de la professionnalisation du métier de l'aide à domicile (Puissant, 2006 ; Devetter, Rousseau, 2007), interroge la nature même de ces activités de services. Elle s'exprime concrètement par une tendance à une "rationalisation professionnelle » (Gadrey, 1994 ; Ughetto, op.cit.), qui se manifeste par une parcellisation des tâches, une intensification des rythmes de travail, si bien que, de plus en plus, les relations privilégiées des aides à domicile avec les usagers laissent la place à des « défilés » d'intervenantes chez les personnes. Nous y reviendrons.

L'externalisation et la rationalisation des processus de production sont largement mobilisées pour légitimer cette industrialisation.

\section{L'externalisation des fonctions assure-t-elle la professionnalisation?}

C’est, en économie industrielle, un point relativement consensuel : le recours croissant à l'externalisation de fonctions de services (bâtiment, maintenance, gestion de personnels) accompagne certaines formes de professionnalisation. Mais ces processus d'externalisation sont efficaces lorsqu'ils s'opèrent, d'une part à travers des logiques techniques de substitution de tâches pour des fonctions périphériques, d'autre part dans des logiques où l'externalisant (ici le ménage, l'aidant familial, la famille) se centre sur son cœur de métier. Or quel est le « cœur de métier » de l’unité ménage, lorsque l'externalisation concerne par exemple le soin à ses (beaux)-parents vieillissants ? Pourquoi la formule selon laquelle « une société qui s’industrialise est une société qui se professionnalise " est-elle si peu transférable dans le cas de l'aide à domicile ? Est-ce l'organisation du travail, ou l'essence même de ce que l'on tente d'externaliser qui pose problème?
Dans le champ de l'aide à domicile, la reconnaissance de compétences professionnelles semble au contraire freinée par l'idéologie de l'externalisation de l'activité domestique, et par la représentation de la division sexuée des tâches (Bourdieu 1998 ; Méda, Périvier 2007 ; Dussuet 1997). Ces freins renvoient d'abord à la conception selon laquelle les compétences mobilisées dans les services d'aide à domicile seraient de simples prolongements des aptitudes développées dans la sphère domestique, ce que chercheurs (Dussuet, 1997, 2005) et salariés infirment depuis longtemps.

\section{Q. " On dit parfois que ces métiers sont le prolon- gement de ce que l'on fait chez soi ? "}

« Ah non pas du tout, sûrement pas (...) Les filles (...) disent "oui mais j'ai soigné ma vieille tante”, et elles croient que donner un coup de gant de toilette sur le bout du nez, tu sauras être assistante de vie sociale! C'est des filles qu'on ne peut pas embaucher ! » (Aide à domicile, salariée association) (66 ans, ancienne infirmière, 18 ans d'ancienneté).

\section{Rationaliser n'est pas professionnaliser}

\section{- Rationalisation et division du travail}

La dynamique des professions, suivant la logique de la rationalisation, s'est traditionnellement incarnée dans le processus du «dirty work» (sale boulot) : en s'ennoblissant par l'incorporation de nouvelles tâches plus prestigieuses, une profession crée des catégories d'emplois moins qualifiés et, en bout de chaîne, de l'emploi non qualifié. C'est ce qui s'est produit en 1951 lors de la création du statut des travailleuses familiales, devenues en 1999 techniciennes d'intervention sociale et familiale. Pour constituer cette profession, les tâches ménagères ont été éliminées, au moins dans le travail prescrit, mettant à l'honneur les tâches éducatives et d'accompagnement social. Les tâches ménagères étaient effectuées par des "aides ménagères » requalifiées ensuite « aides à domicile ». La dynamique de création du statut d'auxiliaire de vie sociale n'est pas éloignée. L'aide à la toilette revient, en prescription, davantage aux auxiliaires de vie sociale. Nos entretiens montrent cependant que cette distinction dans les classifications est peu compatible avec la prise en 
charge globale des usagers. Dans les pratiques, les intervenants réalisent, quel que soit leur statut, une palette d'activités avec, selon les cas, une dominante soin ou une dominante ménage. Une auxiliaire de vie peut-elle rester indifférente à une incontinence qui nécessite que les sols soient nettoyés ? Un agent à domicile peut-il rester indifférent au malaise d'une personne âgée ? En réalisant une veille sanitaire et sociale, une gestion des urgences, l'employé sort du cadre de son travail prescrit. En prenant en charge des tâches de ménage du domicile, une auxiliaire de vie sociale quitte la stricte fonction de soin.

\section{Q. « Vous n’intervenez que sur la personne? "}

«Non. On fait un peu de nettoyage, cuisine, etc. Tu es obligé de faire l'environnement. Si on a fini les soins et qu'il y a un évier plein de vaisselle, on va faire la vaisselle ou débarrasser. On fait un peu par terre. On fait le lit aussi. »

\section{Q. "C’est écrit dans le contrat ? "}

"C'est plus ou moins dit dans le descriptif. Enfin c'est très vague car on nous dit aide à la personne et environnement. Mais la plupart des gens chez qui on intervient ont aussi des aides ménagères (...). Donc on n'est pas censé faire le ménage. Mais on n’y coupe pas. Si on a des draps dégoûtants, on les met dans la machine à laver et on fait partir la machine ». (Auxiliaire de vie sociale, 48 ans ; 12 ans d'expérience, association).

\section{- Rationalisation, services relationnels et autono- mie dans le travail}

La rationalisation professionnelle s'appuie sur une réorganisation du travail, dans une logique de maîtrise des financements publics. La rationalisation du temps de travail et de la gestion des plannings se manifestent par une parcellisation des interventions. Les plans d'aide deviennent de plus en plus précis et prévoient des interventions d'un quart d'heure, par exemple pour mettre un plat dans le four à microondes. Ce quart d'heure d'intervention prévu, s'il est suffisant pour monter les escaliers jusqu'à la porte d'entrée de la personne, d'entrer, de prendre le plat, de le mettre dans le four, d'attendre qu'il soit chaud, puis de le disposer sur la table avec des couverts, est en revanche insuffisant pour prendre des nouvelles de la personne et s'entretenir avec elle. Ce temps est insuffisant pour gérer les imprévus (une personne à accompagner aux toilettes, à changer, une personne déprimée en attente de la seule visite de la journée). La diminution de la durée des interventions engendre une forte parcellisation des interventions des aides à domicile qui "enchaînent les personnes âgées, comme on fait un marathon ${ }^{5}$ ».

La rationalisation des services d'aide auprès de personnes fragiles est-elle cohérente avec la spécificité de l'organisation de l'aide à domicile ? Dictée par le lieu de production, le domicile des particuliers, l'autonomie dans le travail est quasi intrinsèque à l'activité. S'opère donc un ajustement permanent aux contextes spécifiques de populations en situation de fragilité : ajustement de proximité aux situations d'urgence, aux exigences de l'usager et de ses proches, à l'environnement de travail, chaque domicile ayant ses spécificités (Dussuet, Loiseau, 2007).

Comment cette nécessaire et réelle autonomie estelle compatible avec les procédures de rationalisation? Comment en retour rendre ces procédures, elles-mêmes porteuses d'une division du travail et des tâches, compatibles avec la nécessaire globalité de la prise en charge ?

\section{- Professionnalisation et standardisation des tâches}

La globalité de l'intervention de "prendre soin » s'accommode mal des processus de standardisation qui conduisent à des séquences de travail et des prestations hachées. Dans certains départements, le financement de l'Apa (allocation personnalisée d'autonomie) conduit les acteurs à proposer des séquences de travail d'un quart d'heure : le temps de laver le corps d'une personne âgée. Quel temps est-il laissé à la rencontre, au lien qui se crée dans cette prestation de l'intime? On rejoint ici une question centrale : que produit l'agent de l'aide à domicile, quelle est sa mission ? La perte de la notion de globalité et la domination de la mesure de la prestation par sa durée placent les salariés face à des dilemmes. Une clarification de la représentation des tâches

\footnotetext{
${ }^{5}$ Auxiliaire de vie sociale, 42 ans, travaillant dans la même association depuis 11 ans.
} 
assumées par les aides à domicile est donc nécessaire. Est-ce toutefois possible dans un contexte qui identifie professionnalisation à externalisation, rationalisation et standardisation? Ne faut-il pas au contraire dé-domestiquer les aptitudes, reglobaliser les tâches et resingulariser la relation de service, autant d'éléments constitutifs du métier de l'aide à domicile ? L’héritage de l'économie sociale permetil d'avancer dans cette voie ?

\section{LA PROFESSIONNALISATION, FRUIT DES HÉRITAGES DES ASSOCIATIONS D'AIDE À DOMICILE}

La place historique des associations, en situation de quasi-monopole de l'activité d'aide à domicile jusqu'en 1990, a été déterminante pour organiser les activités et façonner le secteur, en faisant émerger des services partiellement co-construits et non standardisables.

\section{Un service initialement pensé et réalisé sur la base d'une co-production}

Les associations d'aide à domicile (ADD) sont issues de deux mouvements, ouvrier et catholique, intégrés dans la mouvance catholique sociale (Dermenjian, 1991). Elles élaborent ainsi un compromis entre des préoccupations d'ordre civique, visant la promotion de la classe ouvrière (le soutien à domicile se fait par et pour les ouvriers), et des préoccupations d'ordre domestique, visant un idéal de charité chrétienne, dans une relation fortement personnalisée (Dussuet, Loiseau, op. cit.).

S’il est souvent noté que l'héritage catholique est encore perceptible dans les associations d'aide à domicile, l'héritage ouvrier est moins souvent évoqué. Pourtant, la promotion de la participation des ouvriers à la réalisation de services d'entraide est essentielle, et contribue à expliquer la forte compatibilité de l'organisation de ces services avec le statut associatif, permettant la «double qualité » à la fois d' « associé et de participant à l'activité » (Vienney, 1994, p. 48). Cette double qualité est organisée dans le cadre d'une fonction employeur collective, béné- vole, assumée par un conseil d'administration, permettant la participation directe des usagers à l'activité de l'association.

La place privilégiée accordée aux usagers ${ }^{6}$ dans les associations a permis la réalisation d'activités coproduites. La relation d'usage est un point d'ancrage des acteurs associatifs pour tenir et rendre compte de la relation complexe entre salariés et usagers. Elle renvoie à une relation institutionnalisée de négociation et d'adaptabilité conjointe : intervenant comme usager définissent à l'usage l'activité réalisée. En prenant en compte les besoins dans leur pluralité, cette co-construction a contribué à une forme d'incomplétude volontaire des contrats qui entre en contradiction avec la circonscription industrielle précise des outputs de l'activité, ou des missions réalisées : "faire réchauffer un plat », « vérifier la prise de médicaments », « effectuer la toilette ».

\section{L'activité professionnelle comme prolongement de l'activité bénévole et/ou domestique}

L'intégration des salariés à l'organisation s'est traditionnellement faite sur la base d'un continuum entre bénévolat et salariat (Demoustier, Rousselière, 2005). Le salariat était alors plutôt synonyme d'autonomie et de polyvalence, et faisait moins référence à la notion de subordination (Demoustier, Ramisse, 2000). Ce continuum était aussi bien utile pour l'association, qui s'assurait un recrutement aisé et l'adhésion de ses salariés, que pour le parcours individuel des personnes, en termes de carrière professionnelle, promotion de l'engagement ou intérêt pour un travail choisi.

L'histoire particulière de la consolidation de l'emploi dans les associations, via le bénévolat, invalide une conception univoque du travail. Les formes d'engagement aux mondes du travail sont multiples (volontariat, salariat, bénévolat, militan-

\footnotetext{
${ }^{6}$ Les associations ont historiquement mis en place un service social, répondant à des besoins d'usagers, et non un service commercial, répondant à des demandes de clients. En effet, les services se sont avant tout développés, non pour répondre à des demandes d'aide individuelles, mais dans un souci plus large de lutte contre la misère des personnes âgées et des mères de famille, sur un territoire. Les enjeux de cohésion sur un territoire, d'égalité, d'émancipation ouvrière (les services étaient pensés par et pour les ouvriers) ont été à l’origine de la création des premières associations.
} 
tisme) et l'association représente un lieu privilégié d'observation de leurs relations. Les frontières entre ces différentes formes ne sont pas étanches. Le registre d'action domestique, qui privilégie la dimension relationnelle, l'ajustement mutuel et la valeur de fidélité, peut accompagner le passage du bénévolat au salariat, sans pour autant s'estomper. Le registre d'action professionnel, qui s'attache à la dimension des qualifications et des compétences objectivées, peut exister dans un contexte où domine le bénévolat, et peut même en tirer parti. Ainsi, bien que quasi exclusivement salarié, le monde du travail des intervenantes à domicile dans les associations est pluriel.

"Quand ma fille est morte, j'ai décidé que je voulais me consacrer aux autres, sortir un peu de chez moi, de mon malheur. Mes petites mémés, c'est comme mes grands-mères. Mon travail, je veux le faire comme je l'ai toujours fait. Je sais que je le fais bien, car j'aime ça. Les nouvelles, elles, ce n'est pas pareil. Elles aiment moins les personnes âgées, je pense. Elles sont toujours dans le désaccord, dans les revendications. Moi, je n'ai pas envie de parler avec elles, je veux continuer à aller voir mes personnes âgées, tranquille ». (Auxiliaire de vie sociale et déléguée syndicale, 62 ans, travaillant pour la même association depuis vingt ans).

Ainsi, les aides à domicile sont progressivement devenues des bénévoles indemnisées puis rémunérées, jusqu'à devenir salariées de l'association. D’autres associations, comme celles créées dans le cadre des mouvements familiaux populaires, ont parfois salarié juste après la guerre les intervenantes à domicile et, dès 1944, ont ouvert des écoles de formation pour les travailleuses familiales. Le continuum est ici symbolique : bien que d'emblée salarié, le travail est d'abord considéré comme domestique au sens où l'on reproduit chez les usagers les mêmes tâches que chez soi, avant d'être "professionnel », au sens où les services réalisés à l'extérieur de son propre domicile détiennent une plus-value par rapport aux activités pour soi et chez soi.

\section{Professionnalisation industrialisante et mise en tension du fonctionnement des associations}

Lorsqu'on aborde les difficultés qu'elles rencontrent dans leur quotidien professionnel, la majorité des aides à domicile énoncent en premier lieu l'organisation de travail, signe de tensions importantes dans la relation de travail, en particulier du fait de tentatives d'instauration d'une professionnalisation à dominante industrielle. On note dans ce contexte, d'une part une tendance à l'augmentation de la quotité du travail prescrit, et d'autre part une tendance à la complétude des plans d'aide dans le cadre de l'Apa, qui prévoient des temps d'intervention de plus en plus restreints et fragmentés, avec des prescriptions de plus en plus précises, prescriptions qui, souvent, n'intègrent pas les temps de discussion et de socialisation inhérents au travail dans les services relationnels.

Ces éléments entrent en contradiction avec les héritages de l'ESS. D'une part, avec la disparition de l'incomplétude volontaire, la coproduction continue du service rendu avec l'usager est mise en question au profit de la prescription du travail. Contradiction également avec le caractère personnel que requiert toute relation dans des domiciles privés pour des prestations de vie quotidienne auprès de publics fragiles. Ces tensions constituent un frein à l'émergence d'une professionnalité négociée (voir infra) qui serait respectueuse des logiques domestique et civique promues par l'économie sociale.

Pour dépasser ces tensions, n’y a-t-il pas nécessité d'identifier et de reconnaître préalablement la pluralité des logiques de professionnalité effectivement à l'œuvre?

\section{LA DIVERSITÉ DES CONVENTIONS DE PROFESSIONNALITÉ}

Tentons de répondre à cette question à partir des résultats d'une recherche récente (Devetter et alii, 2008). Dans le prolongement des travaux de la sociologie de la justification (Boltanski et Thévenot, 1991), les auteurs ont construit une classification des 
éléments d'entretiens recueillis en trois types d'espaces où opèrent les acteurs : l'espace du besoin, de l'organisation, de l'action publique et collective (Ribault, 2008).

\section{Des professionnalités}

Dans l'espace du besoin, les protagonistes centrent leur action sur la qualité du service dans ses dimensions individuelles (viser l'autonomie de la personne) aussi bien que collectives (soulager les proches), subjectives (être proche, être présent) aussi bien qu'objectives (respecter des normes d'hygiène) ; l'espace de l'organisation est centré sur la performance : performances individuelles (efficacité du salarié dans les gestes effectués), collectives et de l'organisation (ponctualité, gestion des remplacements, adéquation des salariés à l'usager, etc.) ; l'espace de l'action publique et collective est quant à lui centré sur la régulation et la production d'incitations : incitations à consommer, à embaucher, à se fédérer, à négocier, à professionnaliser, à l'égalité. S'opère dans chacun des espaces un processus d'optimisation : l'espace du besoin est le «lieu» d'une recherche de la qualité de service tenant compte de contraintes liées notamment au prix des prestations, mais aussi aux limites d'accès aux informations détenues sur le produit-service luimême et sur l'organisation qui le fournit.

De son côté, l'espace de l'organisation cherche à optimiser son processus de production en conformité avec ses ressources pour aboutir à la définition d'un coût et d'une dépense (pour les familles), qui soit le reflet de ce qu'elle estime être le point d'équilibre de sa viabilité économique. Dans l'espace de l'action publique et collective, les protagonistes tentent quant à eux de légitimer leur intervention sous mandat en générant des effets de régulation ou d'incitation qui concernent aussi bien l'espace du besoin (à travers le subventionnement de la demande par exemple) que dans celui de l'organisation (à travers par exemple la mise en place et le contrôle des procédures d'agrément). Ainsi, dans l'espace du besoin, les acteurs visent à produire un service qui fait du bien à l'usager (et à son entourage); dans l'espace de l'organisation, ils visent à produire un service performant (rapport coût/prix/qualité) ; et dans l'espace de la régulation, ils visent à produire un service juste (justement accessible, choisi et produit).

Chaque type d'acteurs n'est pas en scène dans un unique espace: par exemple, les utilisateurs sont aussi bien «mobilisés » dans l'espace du besoin (ils donnent un avis dans le plan de soins les concernant), que dans ceux de la régulation et de l'organisation (les personnes âgées identifient parfois comment le travail d'aide pourrait être réalisé). Dans chaque espace se joue donc une situation où sont impliqués plusieurs acteurs. Dans l'espace du besoin, les utilisateurs conçoivent ce qu'ils considèrent comme central, en interaction avec ce que les fournisseurs de services, les instances de régulation et les salariés estiment de leur côté comme relevant du «vrai » besoin. C'est donc dans les interactions entre les trois espaces que sera configuré un type de professionnalisation capable ou pas de générer un cercle vertueux entre qualité de service et qualité d'emploi. Chaque espace est lui-même le fruit d'une structuration fondée sur un dialogue, qui peut être une tension c'est-à-dire une confrontation et conjugaison de logiques - entre des acteurs. Dans chacun des trois espaces s'opèrent ainsi des compromis plus ou moins collaboratifs entre les acteurs, qui aboutissent à la définition de ce qu'est la « vraie » qualité du service, le «vrai » coût du service en relation avec son « juste » prix, et de ce qu'est la « bonne » et « juste » régulation.

L'analyse des discours permet de mettre en évidence une diversité des figures de la professionnalisation, que nous avons appelées conventions de professionnalité, mobilisées par chacun des acteurs. Une convention de professionnalité est une logique qui est communément mobilisée - donc partagée - par des acteurs différents (ou un acteur donné), relativement à un espace donné (ou à plusieurs espaces), et qui attribue à la définition de la profession une caractéristique dominante. On aboutit à huit conventions de professionnalité : dans le discours des acteurs, c'est tour à tour, la vocation, l'éthique, le pragmatisme, la rationalisation industrielle, le marché, le politique, la réglementation, la relation de service, ou souvent encore une combinaison de ces conventions, qui va fonder la professionnalité. Le tableau 1 en résume les caractéristiques. 


\section{Tableau 1 \\ Les conventions de professionnalité}

\begin{tabular}{|c|c|}
\hline Vocationnelle & $\begin{array}{l}\text { Capacité à apporter une réponse aux besoins des personnes sur la base de leur expérience, de } \\
\text { l'acquisition informelle de savoirs (auto-formation), de leur amour du métier. Individualisation, voire } \\
\text { naturalisation des compétences: référence aux " qualités humaines et comportementales » fondées } \\
\text { sur l'expérience personnelle du salarié. }\end{array}$ \\
\hline Éthique & $\begin{array}{l}\text { Capacité à générer de l'autonomie chez les personnes, et attitude compassionnelle vis-à-vis d'elles. } \\
\text { Variante plus collective : référence explicite à l'humain plutôt qu'à la personne (être utile à la société } \\
\text { autant qu'à une personne). }\end{array}$ \\
\hline Pragmatique & $\begin{array}{l}\text { Capacité à s'adapter à l'imprévu, à susciter de la confiance à travers la ponctualité et la continuité } \\
\text { de son intervention, à bricoler des solutions mettant en jeu des aptitudes psychologiques et d'écoute. } \\
\text { Prendre en compte les particularités des personnes et des besoins et considérer ce qui n'est pas } \\
\text { prévisible et ce qui n'est pas standardisable. } \\
\text { Efficacité d'intervention au sens de s'efforcer d'apporter des solutions là où se posent des problèmes } \\
\text { dont la résolution est considérée comme impérative. }\end{array}$ \\
\hline Industrielle & $\begin{array}{l}\text { Appel à la possibilité de poser des limites entre les sphères dites "personnelle " et } \\
\text { "professionnelle ". Rompre l'isolement des salariés en créant des collectifs de travail. Ancrer le } \\
\text { professionnalisme dans les compétences et les qualifications. } \\
\text { Penser la figure du professionnel en termes de rationalisation des processus de production et de } \\
\text { division des tâches. } \\
\text { En appeler à la sécurisation des emplois et des parcours professionnels comme condition de la } \\
\text { professionnalité (cf. démarches de certification). La professionnalisation est le fruit d'une ingénierie } \\
\text { technique (cf. appel au développement de labels qualité). }\end{array}$ \\
\hline Marchande & $\begin{array}{l}\text { Capacité à réagir, à s'adapter, à servir en continu, à être proche de l'usager, à identifier ses besoins } \\
\text { dans ses multiples dimensions. Capacité de l'organisation à induire des effets d'image et à créer une } \\
\text { identité d'entreprise. } \\
\text { Penser en termes de rencontre entre offre et demande, et de capacités à " se battre avec les mêmes } \\
\text { armes que la concurrence ». Professionnalité fondée sur la capacité à sélectionner les bons offreurs } \\
\text { des mauvais, à rendre le marché plus transparent et à respecter la liberté des clients en matière de } \\
\text { choix des prestataires. }\end{array}$ \\
\hline Politique & $\begin{array}{l}\text { Mobilisation positive : "la France est en retard " et « il y a un fort potentiel d'emplois » qui est mal } \\
\text { exploité. } \\
\text { Mobilisation critique : caractère propagandiste des effets d'annonce gouvernementaux, flou des } \\
\text { objectifs visés par les décisions de politique publique, effets pervers des outils créés, comme le } \\
\text { chèque emploi service universel, inefficacité des dispositifs d'intermédiation tels que les enseignes. } \\
\text { Vision de la professionnalité qui repose sur une garantie publique et régulée de la qualité des } \\
\text { prestations. } \\
\text { Le territoire continue d'avoir un sens et la professionnalité consiste aussi à garantir un accès équitable } \\
\text { aux services. } \\
\text { Défense du développement des emplois de services coûte que coûte versus la professionnalisation } \\
\text { qui doit viser avant tout la qualité des prestations, la fiabilité du contrôle de cette qualité, ainsi que la } \\
\text { garantie de prise en charge financière de la construction de la qualité par les pouvoirs publics eux- } \\
\text { mêmes. }\end{array}$ \\
\hline Réglementaire & $\begin{array}{l}\text { Définition de la professionnalité fondée sur le respect des règles relatives à la délimitation du } \\
\text { périmètre d'intervention des salariés: ils sont des professionnels puisque des règles existent. } \\
\text { Présence ou pas de conventions collectives. }\end{array}$ \\
\hline Servicielle & $\begin{array}{l}\text { Assimilable à une convention de "rationalisation professionnelle " (Gadrey, 1994). Renvoie d'une } \\
\text { part à la volonté de typifier les cas, de formaliser des méthodes dans un objectif de gain d'effica- } \\
\text { cité des procédures du travail professionnel, à la fois au sens du gain de temps et de la qualité de } \\
\text { la réponse. Renvoie aussi à l'affirmation de la singularité des services d'aide à domicile et à } \\
\text { l'importance accordée à leur valeur relationnelle : " on ne travaille pas à la chaîne ". }\end{array}$ \\
\hline
\end{tabular}




\section{Des vertus de la pluralité} des conventions de professionnalité

Aucune convention de professionnalité ne peut prétendre garantir à elle seule et simultanément la qualité du service rendu à la personne âgée, et la qualité d'emploi (durée du travail, variabilité des temps de travail, niveau de salaire, etc.) de la salariée qui en a la responsabilité (Devetter et alii, 2009). Cela signifie que vouloir asseoir la professionnalisation de l'aide à domicile sur une unique convention reviendrait à nier l'existence des autres dimensions de la qualité de service et d'emploi dont les autres conventions sont porteuses. Autrement dit, l'hégémonie d'une convention peut être dommageable à la qualité de service et d'emploi qu'encourage une autre convention. La professionnalité est donc un construit évolutif en tension, et seule cette tension permet de réguler qualité de service et qualité d'emploi. Les relations qui se tissent entre les conventions affaiblissent tout discours qui tendrait à rendre inéluctables les évolutions du secteur. Donnons-en une illustration à partir des conventions marchande et politique.

Du côté marchand, le diagnostic de la qualité de service repose surtout sur des qualités relatives à l'organisation : il s'agit de s'approcher au plus près des demandes identifiées. À la différence des besoins, les demandes portent sur les modalités de prestation, plus que sur le contenu même de la prestation. Ainsi, on identifie une demande de souplesse d'utilisation, d'adaptabilité, de continuité, de réactivité : être là au bon moment. À cette approche de la qualité de service répond une représentation de l'emploi fondée sur la flexibilité, la capacité adaptative - qui se nourrit d'ailleurs très bien de la convention vocationnelle ( « aimer son métier »), tout comme de la convention pragmatique («faire plein de choses »), voire de la convention éthique («aimer les autres ») -, et sur la construction d'une identité d'entreprise plutôt que d'une identité professionnelle. Il n'y a plus lieu de distinguer les services à partir de la nature juridique des intervenants : seule compte la capacité d'écoute et de réponse. Le client devient employeur et le salarié devient entrepreneur de lui-même. Cette conception prône une transparence des transactions et transforme de ce fait la professionnalité en boîte noire. Enfin, cette convention mobilise la notion de libre choix : choix de l'usager entre le soin à domicile et le soin en établissement, choix du client en matière de type de prestataires (gré à gré, mandataire, prestataire), ou encore choix du type d'agrément ou régime d'autorisation auquel se soumet l'intervenant.

La convention de professionnalité est qualifiée de politique lorsque les logiques mobilisées dans les discours des acteurs relativement à ce qu'est pour eux une profession, s'inscrivent dans un registre d'actions publiques visant soit à développer l'emploi, soit à gérer des (dés)équilibres budgétaires, soit encore à lutter contre des inégalités d'accès ou territoriales. À l'exception de ceux relatifs à la création d'emploi à tout prix, la plupart des discours tenus par les différents acteurs dans le cadre de cette convention de professionnalité rappellent les limites de la convention marchande du point de vue de ses conséquences tant sur la qualité de service que sur la qualité d'emploi.

Par exemple, la trans-territorialité des structures intervenantes que suppose la convention marchande (l'idée qu'une prestation peut être rendue de manière identique quel que soit le territoire d'intervention) est en tension forte avec le caractère affirmé que prend le territoire dans la convention politique. L'ancrage territorial des besoins constitue dans cette dernière une des dimensions de la qualité de service, d'autant qu'il renvoie à un ancrage territorial de la solvabilité de la demande et donc à une dimension de justice du point de vue de l'accès aux services.

Alors que la convention marchande a tendance à se référer à une professionnalité sui generis, la convention politique redonne aux acteurs un espace de dialogue, de co-construction et de négociation apte à définir la qualité. Cet espace situe la professionnalité en son centre, considérant que la qualité de service en découle. Dans cette convention, les conditions d'emploi et de travail font l'objet d'une attention particulière : d'où le souci de construire des emplois du temps consolidés, de former les personnels à tous les niveaux, d'assurer des passerelles entre métiers ou encore d'encourager la transmission des savoirs. En lien plus direct avec la qualité de service, la convention politique est en tension forte avec la convention marchande, notamment sur le rôle que 
cette dernière attribue au prix en tant que pivot de la rationalité des agents. Si la convention politique n'est pas anti-marchande, elle tend à considérer le prix comme inséparable de la qualité de service d'une part, et de la qualité d'emploi d'autre part. La formule "se méfier des prix bas" en est un bon reflet en renvoyant à l'idée que les dépenses de formation font partie des coûts et doivent être répercutés, quitte à mettre en place un subventionnement de la demande ou de l'offre. Enfin, la co-construction de la qualité de service et d'emploi passe, dans le discours de la convention politique, par l'instauration de procédures éprouvées de contrôle de l'activité, ce qui ici aussi est en tension mais n'est pas incompatible avec la convention marchande.

Réduire la professionnalisation à un processus d'individualisation de la qualification des salariés et à une démarche de rationalisation et de standardisation des prestations contribue à brouiller la représentation des tâches assumées par le métier d'aide à domicile. Les effets de ce brouillage sont néfastes pour la qualité de l'emploi comme pour celle du service alors que la pluralité des formes d'engagement dans le travail des intervenantes à domicile constitue précisément une caractéristique majeure du champ des organisations relevant de l'économie sociale.

Nous avons mis au jour la diversité réelle des manières d'être professionnel dans le métier d'aide à domicile, diversité à laquelle les organisations de l'économie sociale sont accoutumées de par leur histoire, faite d'injonctions et d'évolutions contradictoires.

Parvenir à des situations où la professionnalisation ne s'oppose plus à la qualification collective des individus, ne requiert-il pas comme préalable une reconnaissance négociée et instituée de la pluralité des professionnalités? Cette reconnaissance, en contribuant à dé-domestiquer les aptitudes, à reglobaliser les tâches et à resingulariser la relation de service, pourrait recentrer la valeur des services autour d'une valeur d'usage dont la maximisation s'effectuerait sous contrainte de la minimisation de la valeur d'usure des ressources mobilisées pour la produire. La dé-domestication signifie ne plus considérer le travail de l'aide à domicile comme une externalisation des fonctions domestiques. La reglobalisation renvoie à la remise en question de la frontière entre les tâches nobles et le dirty work (sale boulot), frontière qui résulte de la parcellisation du travail dans un but unique de performance économique. Enfin, la resingularisation fait référence à la réalité selon laquelle il n’y a jamais deux relations identiques dans le métier de l'aide à domicile.

Restituer la pluralité des formes de professionnalité mobilisées dans l'exercice du métier d'aide à domicile ne permettrait-il pas de redonner aux organisations relevant de l'économie sociale et solidaire l’opportunité d'un nouvel élan? Cet élan serait appelé à se fonder sur une redéfinition de l'utilité sociale des services d'aide autour de l'accessibilité des services, de la soutenabilité des emplois - c'està-dire des emplois décents et reconnus - et de la qualité des prestations.

\section{Bibliographie}

Aballea F. (2005), « La professionnalisation inachevée des assistantes maternelles », Recherches et Prévisions, CNAF, $\mathrm{n}^{\circ}$ 80, Juin.
Artis A., Demoustier D., Hofmann B., Puissant E., (2008), Économie sociale et solidaire et régulations territoriales, Rapport pour la DIIESES. 
Bisault L., Destival V., Goux D. (1994), « Emploi et chômage des non-qualifiés en France », Économie et Statistique, $\mathrm{n}^{\circ} 273$, Insee.

Boltanski L., Thevenot L. (1991), De la justification. Les économies de la grandeur, Gallimard, 480 p.

Bourdieu P. (1998), La domination masculine, Paris, Le Seuil.

Chardon O (2002), «La qualification des employés », Insee, Série des Documents de travail, $\mathrm{n}^{\circ}$ F0202, Mars.

Chenu A., Burnod G. (2001), « Les employés qualifiés et non qualifiés : une proposition d'aménagement de la nomenclature des catégories socioprofessionnelles », Travail et Emploi, Dares, Avril.

Demoustier D., Ramisse M.-L. (2000), L'emploi dans l'économie sociale et solidaire, Arles, ThierryQuinqueton éditeur.

Demoustier D., Rousseliere D. (2005), L'économie sociale dans la structuration des activités de services : vers un dépassement des contradictions de l'organisation capitaliste du travail ?, Grenoble.

Dermenjian G. (1991), «Les femmes dans les mouvements familiaux populaires de 1935 à l'aprèsguerre ", in Femmes, familles et action ouvrière : pratiques et responsabilités féminines dans les mouvements familiaux populaires (1935-1958), Villeneuve d'Ascq, Les Cahiers du GRMF, $\mathrm{n}^{\circ} 6$, pp. 35-58.

Devetter F.-X., Fraisse L., Gardin L., Jany-Catrice F., Gounouf M.-F., Ribault T. (2008), L'aide à domicile face aux services à la personne: mutations, confusions, paradoxes, Rapport pour la DIIESES, mars.

Devetter F.-X., Jany-Catrice F., Ribault. (2009), Les services à la personne, La Découverte, collection « Repères ", Paris.

Devetter F.-X., Rousseau S. (2007), « Services domestiques : quelles perspectives pour une stratégie d'industrialisation? ", Revue d'Économie Industrielle, $\mathrm{n}^{\circ} 119$, $3^{\mathrm{e}}$ trimestre.

Dussuet A. (1997), « Domination et dévalorisation dans les rapports sociaux de sexe", in Nouveau millénaire, Défis libertaires, septembre. http:// 1libertaire.free.fr/ADussuet01.html

Dussuet A., Loiseau D. (2007), « Les services aux familles offertes par les associations : un modèle de service "entre" formel et informel ? ", in Dussuet A., Lauzanas J.-M., L'économie sociale entre informel et formel : paradoxes et innovations, Presses Universitaires de Rennes, Rennes.

Friedmann G. (1976), Le travail en miettes : spécialisation et loisirs, Paris, Gallimard.

Friedmann G., Naville P. (1962), Traité de sociologie du travail, Paris, A. Colin.

Gadrey J. (1994), «La modernisation des services professionnels - Rationalisation industrielle ou rationalisation professionnelle? », Revue française de sociologie, XXXV, pp. 163-195.

Gadrey N., Jany-Catrice F., Pernod-Lemattre M. (2004), « Les conditions de travail des employés non qualifiés ", in Méda D., Vennat F., Le travail non qualifié, permanences et paradoxes, La Découverte, Paris, pp. 182-196.

Jany-Catrice F., Ribault T. (2007), « Les services à la personne, des inégalités inévitables », in Batifoulier, Ghirardello, De Larquier Remillon, Approches institutionnalistes des inégalités en économie sociales, tome 2 : Politiques, L'Harmattan.

Lallement M. (2007), Le travail : une sociologie contemporaine, Paris, Gallimard.

Méda D., Vennat F. 2004 (sous la dir. de), Le travail non qualifié, permanences et paradoxes, La Découverte, coll. « Recherches, » 420 p.

Méda D., Perivier H. (2007), Le deuxième âge de l'émancipation: la société, les femmes et l'emploi, Paris, Le Seuil. 
Naville P. (1956), Essai sur la qualification du travail, Paris, M. Rivière.

Oiry E., D’Iribarne A. (2001), «La notion de compétence : continuités et changements par rapport à la notion de qualification ", Sociologie du travail, Volume 43.

Puissant E. (2006), Associations d'aide à domicile : des tensions à l'œuvre dans la relation de travail, Contribution au colloque annuel de l'Association d'Économie Sociale, Paris. economix.u-paris10.fr/ pdf/colloques/2007_AES/puissant.pdf

Puissant E. (2008), Évolutions et enjeux des services à la personne: les associations iséroises d'aide à domicile, Lyon, Les publications de l'ADEES Rhône-Alpes, Mai.

Reynaud J.-D. (2001), «Le management par les compétences: un essai d'analyse ", Sociologie du travail, Volume 43.

Ribault T. (2007), « Des services qui font du bien », in L'économie des services pour un développement durable, Colloque de Cerisy, 14-21 juin 2006, éd. L’Harmattan, Paris.
Ribault T. (2008), « Aide à domicile : de l'idéologie de la professionnalisation à la pluralité des professionnalités ", Revue Française de SocioÉconomie $\mathrm{n}^{\circ} 2$.

Rose J. (2004), «Travail sans qualité ou travail réputé non qualifié ? ", in Méda D., Vennat F., Le travail non qualifié, permanences et paradoxes, La Découverte, Paris, pp. 227-254.

Simonet-Cusset M. (2004), "Penser le bénévolat comme travail pour repenser la sociologie du travail », Revue de l'IRES, n 44, janvier.

Ughetto P. (2002), « Compétence de service : État des lieux d'une problématique », Document de travail de l'IRES nº 02/03.

Vasselin K. (2002), « Faire le ménage : de la condition domestique à la revendication d'une professionnalité », in Piotet F., La révolution des métiers, PUF.

Vienney C. (1994), L'économie sociale, Paris, La Découverte.

Weber A. (2006), « Regard sur l’APA, trois ans après sa création », Données sociales, la société française, édition 2006, pp. 603-611.

\section{Résumé}

\section{Associations d'aide à domicile : pluralité des héritages, pluralité des professionnalités}

Florence Jany-Catrice, Emmanuelle Puissant, Thierry Ribault

Dans les discours des acteurs de l'aide à domicile, le terme de professionnalisation a supplanté celui de qualification. Alors que la qualification reposait sur une reconnaissance collective des savoir-faire des salariés, la professionnalisation n'est pas consensuelle, et recouvre des significations très différentes. Pour répondre à la diversité des héritages de l'économie sociale, qui se manifeste par des formes multiples d'engagement dans le monde du travail, nous mobilisons plusieurs conventions de professionnalité : pour être effective, la professionnalisation dans les associations d'aide à domicile doit être reconnue dans sa pluralité.

Mots clés

Aide à domicile, professionnalisation, savoir professionnel

Journal of Economic Literature: J 44, J 24 